\title{
Uso de um jogo de tabuleiro na reabilitação dos membros superiores de idosos institucionalizados em Portugal: um estudo piloto quase-experimental
}

\author{
Board game for the upper limbs rehabilitation in \\ institutionalised elderly from Portugal: a quasi- \\ experimental pilot study
}

\author{
Marlene Neves Rosa' 1 \\ Sara Gordo² \\ Ricardo Pocinho 3 \\ Raquel Marinho 4 (1)
}

\footnotetext{
1-2Escola Superior de Saúde, Instituto Politécnico de Leiria; ciTechCare - Center for Innovative Care and Health Technology (Leiria). Portugal. marlene.rosa@ipleiria.pt, saraoliveiragordo@gmail.com ${ }^{3}$ Centro Interdisciplinar de Ciências Sociais, Escola Superior de Educação e Ciências Sociais do Politécnico de Leiria (Leiria). Portugal. ricardo.pocinho@ipleiria.pt ${ }^{4}$ Instituto Politécnico de Leiria (Leiria). Portugal. raquel.vilar.marinho@hotmail.com
}

RESUMO | INTRODUÇÃO: A utilização do jogo como estratégia de reabilitação tem revelado um especial impacto nas variáveis cognitivas no idoso, não sendo consensual o seu impacto em indicadores físicos. OBJETIVO: Medir o efeito do treino com um jogo de tabuleiro na meIhoria da coordenação dos membros superiores e na força de preensão palmar em idosos institucionalizados. Foi também caracterizada a percepção dos idosos sobre o jogo como estratégia de reabilitação. MATERIAIS E MÉTODOS: Foi conduzido um estudo quase-experimental, longitudinal, incluindo 10 idosos institucionalizados sem alterações significativas na cognição (6CIT 0-7) e na mobilidade dos membros superiores. Este grupo foi inicialmente avaliado (T0) quanto à Força de preensão manual (FPM) e quanto à coordenação dos membros superiores (Bateria de testes EUROFIT), repetindo a avaliação após 2 semanas de terapias convencionais ( $\mathrm{T} 1)$, tendo sido novamente reavaliados 2 semanas após frequentarem as terapias convencionais mais 2 horas adicionais de um programa com um jogo de tabuleiro (T2). Foi ainda coletada a perceção dos idosos sobre a experiência com o jogo. Foi comparada a evolução entre T0-T1 e T1-T2 usando o teste de Wilcoxon. RESULTADOS: Apenas entre T1 e T2 ocorreram mudanças significativas na EUROFIT ( $p=0.005)$ e na FPM para ambos os membros ( $p=0.005 ; p=0.007)$. Os idosos destacaram uma maior relevância do jogo no trabalho em equipe, no estímulo de raciocínio e de agilidade dos membros superiores. CONCLUSÃO: O jogo de tabuleiro é uma potencial ferramenta para completar a terapia convencional, sendo a experiência considerada muito positiva pelos idosos participantes (ClinicalTrials.gov IDIPL10062019).
ABSTRACT | INTRODUCTION: Using games as a rehabilitation strategy has significantly impacted cognitive variables in the elderly; however, its impact on physical indicators is not consensual. OBJECTIVE: To measure the effect of a training program with a board game on upper limb coordination and handgrip strength of institutionalized elderly. The elderly's perception of playing a board game as a rehabilitation strategy was also characterised. MATERIALS AND METHODS: A longitudinal quasi-experimental study was carried out, including 10 institutionalized elderly people without significant changes in cognition (6CIT 0-7) and upper limb mobility. This group was initially evaluated (TO) for handgrip strength (HGS) and coordination of the upper limbs (EUROFIT test battery), repeating the assessment after 2 weeks of conventional therapies ( $\mathrm{T} 1$ ), and also again 2 weeks after attending conventional therapies plus an additional 2 hours of a board game program (T2). The perception of the elderly about their experience with the game was also collected. The evolution between T0-T1 and T1-T2 was compared using the Wilcoxon test. RESULTS: Only between $\mathrm{T} 1$ and $\mathrm{T} 2$ were significant changes in EUROFIT $(p=0.005)$ and HGS for both members $(p=0.005$; $p=0.007$ ). A greater relevance of game-based program for teamwork, stimulating reasoning, and agility of the upper limbs was perceived. CONCLUSION: The board game is a potential tool to complement conventional therapy, and the experience is well perceived by the elderly participants (ClinicalTrials.gov IDIPL10062019).

KEYWORDS: Aged. Recreation therapy. Upper extremity. 


\section{Introdução}

O envelhecimento populacional é uma realidade mundial, sendo que Portugal se destaca como um dos países com valores mais elevados na Europa. É esperado que Portugal seja, em 2050, o quarto país da União Europeia com maior percentagem de idosos (25\%). ${ }^{1}$ O envelhecimento da população em Portugal caracteriza-se por elevados níveis de institucionalização, sendo que as estimativas apontam para um universo de mais de 55000 idosos a viver em mais 1500 lares, o que representa $3.2 \%$ das pessoas idosas. 2 Para ajudar a caracterizar este cenário, é importante esclarecer que $85 \%$ destes residentes têm mais de 75 anos de idade ${ }^{2}$, mostrando-se uma população extremamente envelhecida. Dados de caracterização da população idosa em Portugal revelam ainda uma significativa incapacidade da população com 65 ou mais anos. Neste contexto, cerca de $50 \%$ desta população revela ter muita dificuldade ou não conseguir realizar, pelo menos, uma entre as atividades da vida diária (AVDs). $\stackrel{3}{ }$ Por exemplo, cerca de $14 \%$ apresentam dificuldade em tomar banho/ vestir-se, atividades altamente dependentes da função dos membros superiores. $\frac{3}{}$

A reabilitação regular é benéfica para as habilidades funcionais da pessoa idosa, ajudando a manter a independência na realização das AVD's. ${ }^{4}$ Os processos de reabilitação convencionais têm demonstrado resultados significativos na pessoa idosa, em ganhos de força, como em melhoria de mobilidade e coorde-

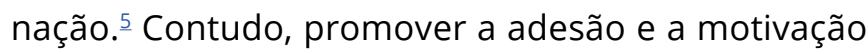
desta população em processos longos de reabilitação é um verdadeiro desafio. ${ }^{6}$ Com vista a melhorar o nível de motivação durante a reabilitação, a escoIha de estratégias deve focar-se em atividades que promovam desafio, divertimento e socialização. ${ }^{\beth}$ Por exemplo, é consensual que os programas de reabilitação, quando suportados por atividades recreativas, aumentam o potencial de reabilitação a longo prazo.. A propósito, a utilização de jogos ou componentes de gamificação tem sido aceita como um método efetivo para melhorar a motivação do idoso e a adesão aos processos de reabilitação. ${ }^{9}$ Apesar da popularidade dos jogos digitais, os jogos de tabuleiro têm demonstrado serem eficazes na promoção de interações pessoais, tendo a capacidade de envolver e motivar a relação com familiares, tutores ou profissionais de saúde, enquanto ocorre aprendizagem (motora, cognitiva, etc.). $\frac{10}{}$
O desenho e implementação adequados de programas de reabilitação com vista à manutenção da funcionalidade do membro superior na pessoa idosa é uma prioridade, e a sua gestão depende da utilização de indicadores de monitorização. Por exemplo, os autores Ibrahim et al. ${ }^{11}$ relatam a monitorização de um plano de intervenção geriátrica com base na apreciação quinzenal dos valores de preensão manual em uma amostra idosa. Em concordância, os déficits de coordenação também são um excelente indicador de funcionalidade e um preditor de alterações cognitivas. ${ }^{12}$

Apesar da importância destes indicadores na funcionalidade da pessoa idosa, a grande maioria dos estudos que implementaram jogos de tabuleiro nesta comunidade tem o seu foco no impacto para as variáveis cognitivas ${ }^{13-15}$, não se conhecendo literatura que valoriza o impacto desta estratégia em importantes indicadores físicos, tais como a coordenação dos membros superiores ou a força de preensão palmar, já referidos. Estes indicadores têm sido amplamente estudados, apenas quando associados à implementação de jogos tecnológicos, nem sempre ajustáveis no custo e no perfil de literacia digital de todos os idosos. ${ }^{16}$ Assim, o presente estudo propõe-se a medir o efeito do treino com um jogo de tabuleiro na coordenação dos membros superiores e na força de preensão palmar em idosos institucionalizados, quando comparado com o efeito isolado de um programa de reabilitação convencional. A hipótese a comprovar é o benefício acrescido do jogo para a melhoria das variáveis em estudo. Adicionalmente, pretende-se caracterizar a percepção dos idosos com o envolvimento no jogo.

\section{Materiais e métodos}

Foi realizado um estudo piloto, quase-experimental, longitudinal de amostragem selecionada por conveniência, respeitando o Standard Protocol Items: Recommendations for Interventional Trials (SPIRIT). Este estudo é parte do protocolo de estudo registado na plataforma ClinicalTrials.gov com o IDIPL10062019 a 6 de novembro de 2019. Foram convidados a participar idosos institucionalizados numa Instituição de Longa Permanência (ILPI) na região centro de Portugal (cidade de Coimbra). Após explicação dos procedimentos do estudo, foram considerados elegíveis idosos que 
(i) aceitaram participar no estudo, (ii) com pontuação de 0-7 no instrumento Six-item cognitive test (6CIT), (iii) e com preservação de mobilidade dos membros superiores (permitindo o alcance horizontal e a simples manipulação de um copo). Foram excluídos idosos com dificuldades graves de visão. Os procedimentos necessários de garantia de anonimato, privacidade e confidencialidade de dados, assim como a obtenção de consentimento informado e voluntariedade de participação no estudo foram assegurados. O estudo foi aprovado em reunião de parecer pela direção da instituição.

\section{Procedimentos}

Todas as avaliações dos participantes foram realizadas por uma única investigadora fisioterapeuta, previamente treinada para a implementação dos instrumentos selecionados. Decorreu um primeiro momento de avaliação dos participantes (T0), seguido de uma reavaliação ao fim de 2 semanas a frequentar terapias convencionais (T1) (Tabela 1). Nas duas semanas seguintes, os mesmos participantes tiveram acesso a mais 2 horas/semana de um Jogo originalmente desenvolvido para o efeito intitulado Jogo das Mãos TA!TI!. Este jogo foi implementado como estratégia complementar às terapias convencionais. As 2 horas por semana de Jogo das Mãos TA!TI! em duplas (2 jogadores de cada vez) foram distribuídas pela equipe multidisciplinar (fisioterapeuta, terapeuta ocupacional, psicomotricista) numa estratégia individualizada, conforme as dificuldades e a motivação de cada dupla de jogadores (Tabela 2). No fim deste período os idosos participantes foram novamente reavaliados (T2). As terapias convencionais, assim como o plano de execução do jogo, estão descritos nas tabelas 1 e 2, respetivamente. 0 processo de acompanhamento dos participantes ocorreu no período de março a maio de 2021.

Tabela 1. Descrição do programa de terapias convencionais a que os participantes do estudo estiveram sujeitos entre T0 e T2

\begin{tabular}{llll}
\hline \multicolumn{2}{l}{ Terapias Convencionais (T0-T2) } & & \\
\hline Descrição & Individual/Grupo & Tempo Sessão & 3 \\
Fisioterapia & Individual & 45 & 3 \\
Treino de Equilíbrio e & Individual & 20 & \\
marcha & & 25 & 2 \\
Classe de mobilidade & Grupo & 45 & 1 \\
Passeio no exterior & Grupo & 60 & 1 \\
Expressão plástica & Grupo & 60 & 1 \\
Jogo do Bingo & Grupo & 20 & 2 \\
Estimulação Sensorial & Individual & 20 & \\
\hline
\end{tabular}

Fonte: Os autores (2021).

O jogo de tabuleiro usado no presente estudo foi o Jogo das Mãos TA!TI!, específico para treino da coordenação e ritmo dos membros superiores, com múltiplos estímulos cognitivos. A escolha deste jogo foi fundamentada pelos seus resultados já conhecidos como medida de performance da função dos membros superiores. ${ }^{17}$ Este jogo apresenta 1 tabuleiro de mesa, um conjunto de cartas de Jogo Simples (para jogar com uma mão); cartas de Jogo Duplo (para jogar em simultâneo com duas mãos); 1 dado para planejar o Jogo Simples; cartas para planejar o Jogo Duplo; 1 Campainha; 1 Copo. Para interagir com o jogo, o tabuleiro deve ser colocado em cima da mesa, centrada com o tronco do jogador. Em primeiro lugar, o avaliador escolhe uma face do dado, que vai instruir o idoso a pronunciar um som a cada posição do copo. É apresentado um conjunto de 5 cartas com símbolos diferentes e diferentes escolhas de posições do copo (Figura 1). 


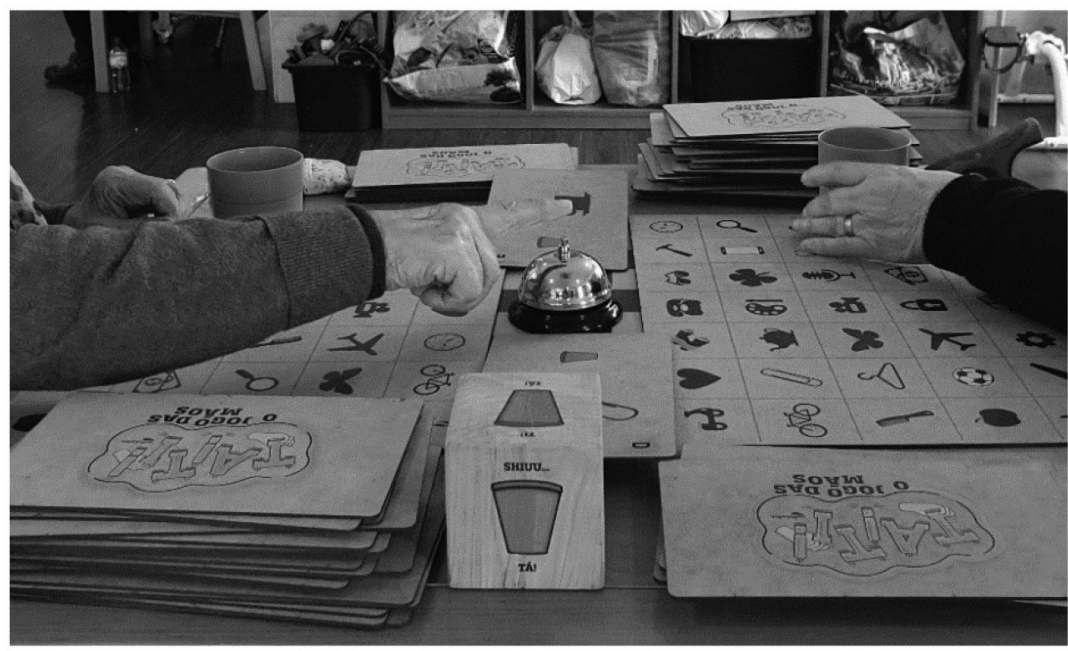

Fonte: Os autores (2021).

Em primeiro lugar, procedeu-se à interação com o Jogo através de uma jogada unilateral. Foi realizado um treino prévio para o idoso compreender as dinâmicas e as regras do jogo. O jogo foi sempre realizado em grupos de 2 jogadores. As cartas do jogo dão informação sobre as figuras a procurar e interagir no tabuleiro, pousando o copo numa posição que também é atribuída em cada jogada. Em conjunto, o idoso deve pronunciar um som que as regras do jogo determinem (TA, TI, SHIU).

O idoso treinou ainda através de uma jogada bilateral. Assim, todo o procedimento anterior foi repetido, mas desta vez recorrendo a cartas de jogo com dinâmicas de interação com o tabuleiro envolvendo os dois membros superiores, enquanto manipulam 2 copos, em posições decididas por cartas de jogo (Figura 2).

Figura 2. Componentes do Jogo das Mãos TA!TI! para o Desafio Bilateral

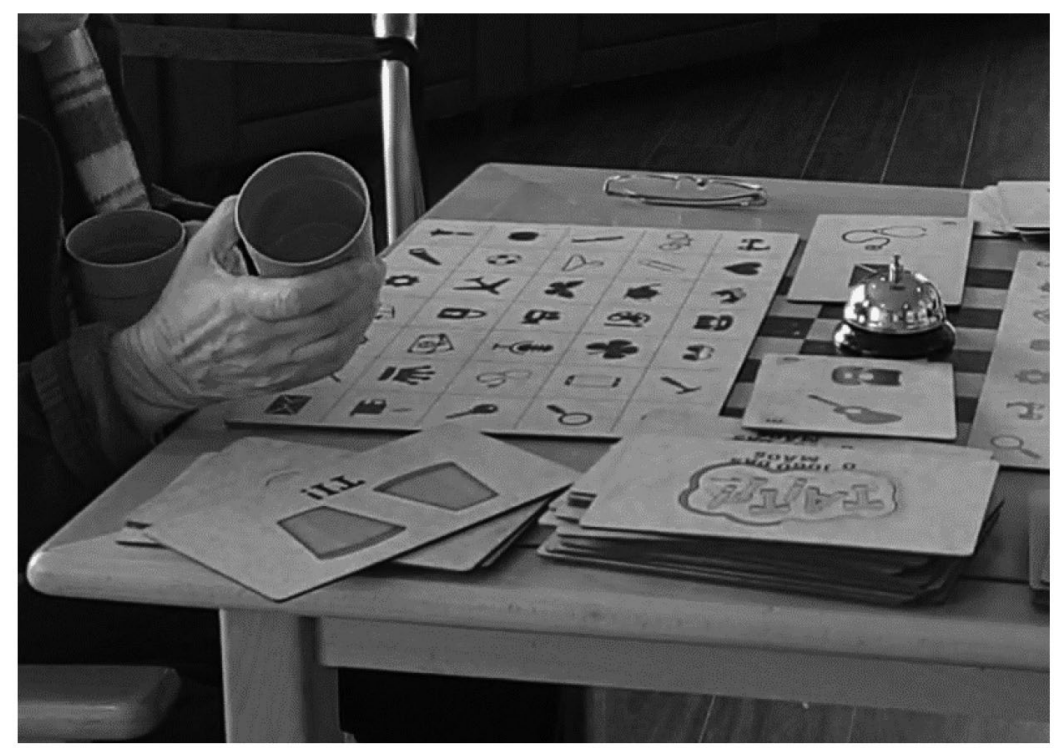

Fonte: Os autores (2021). 
Tabela 2. Descrição do programa com o Jogo das Mãos Tati a que os participantes do estudo estiveram sujeitos entre T1 e T2

\begin{tabular}{lllll}
\hline $\begin{array}{l}\text { Jogo das Mãos Tati (T1-T2) } \\
\text { Duplas de Jogadores }\end{array}$ & $\begin{array}{l}\text { Semana } 1 \\
\text { (min/ss) } \\
\text { Desafio Unilateral }\end{array}$ & $\begin{array}{l}\text { Semana 2 } \\
\text { (min/ss) }\end{array}$ & \\
\hline P1+P2 & 60 & 30 & 60 & 60 \\
P3+P4 & 30 & 30 & 30 & 30 \\
& 30 & 30 & 30 \\
P5+P6 & 30 & 30 & 60 & 60 \\
& 30 & & & 60 \\
P7+P8 & 45 & 30 & 60 & 60 \\
P9+P10 & 45 & 30 & 60 & \\
\hline
\end{tabular}

Fonte: Os autores (2021).

\section{Protocolo de Avaliação}

Dos instrumentos implementados nos participantes em estudo, o questionário de avaliação sociodemográfica foi apenas implementado em T0 e a avaliação das perceções do idoso sobre o trabalho com o Jogo das Mãos TA!TI! foi apenas implementado em T2. A avaliação cognitiva e da coordenação dos membros superiores foi repetida em T0, T1 e T2, na ordem apresentada.

Questionário de avaliação sociodemográfica - Foram coletadas informações sociodemográficas, com base nos seguintes critérios: idade (resposta aberta); sexo (feminino; masculino); estado civil (solteiro, casado, viúvo, divorciado/separado e união estável); escolaridade (não sabe ler/escrever; sabe ler e escrever sem possuir grau de ensino, ensino básico primário, ensino básico preparatório, ensino secundário, ensino médio, ensino superior).

Avaliação Cognitiva - Foi implementado o 6CIT para a avaliação cognitiva dos idosos participantes. O 6CIT é considerado um instrumento sensível e válido para diagnosticar e rastrear alterações de cognição em idosos, demonstrando mais sensibilidade, quando comparado com o Mini-Exame de Estado Mental. 18 O teste consiste em 6 perguntas simples que abordam domínios como a capacidade de localização temporal (ano, mês, dia), memorização de 5 items (nome e endereço), habilidade de verbalizar contagem decrescente (20 até 1) e nominação reversível de meses do ano (dezembro a janeiro), terminando com a avaliação dos seguintes domínios: orientação, aprendizagem, memória e cálculo. ${ }^{19}$ A versão portuguesa deste instrumento demonstrou boa consistência interna e reprodutibilidade. ${ }^{20} \mathrm{~A}$ pontuação deste instrumento é atribuída de forma inversa e as perguntas são ponderadas, sendo que os resultados podem assumir valores de 0 a 28. As pontuações de 0-7 indicam não existirem alterações, enquanto as iguais a 8 ou superiores são consideradas indicativas de comprometimento cognitivo.

Avaliação da coordenação dos membros superiores - A avaliação de coordenação dos membros superiores foi realizada utilizando o teste de toque em discos da bateria de testes de aptidão física do EUROFIT.21 Para operacionalizar o teste, foram colocados sobre a superfície da mesa, dois discos de papel de $20 \mathrm{~cm}$ de diâmetro, fixados horizontalmente a uma distância de $60 \mathrm{~cm}$ (os seus centros estão a $80 \mathrm{~cm}$ um do outro); e uma placa de papel retangular de $10 \times 20 \mathrm{~cm}$ entre os dois discos. A mesa deve ser ajustada, na sua altura, de acordo com a estatura de cada avaliado, devendo ficar à altura da região umbilical do avaliado. Para proceder à avaliação, o avaliado deve ser orientado para se colocar à frente da mesa, em pé, com os pés ligeiramente afastados. O avaliado deve colocar a mão no centro da placa retangular, e com a outra (a mão de preferência do avaliado), efetuar um 
movimento de vai e volta tão rápido quanto possível entre os dois discos, passando por cima da mão fixada na placa retangular. Ao comando de "Pronto... Vai!" do examinador, o avaliado deve executar rapidamente 25 ciclos com a mão, batendo nos dois discos; sem parar antes do sinal "Alto!" do examinador. O examinador conta em voz alta o número de ciclos efetuados. O teste deve ser feito três vezes e o meIhor resultado registado. Algumas regras são fundamentais para garantir a execução adequada do teste, nomeadamente: a mão colocada na placa retangular deve permanecer na mesma posição durante toda a duração do teste; o avaliado deve ter o cuidado de tocar nos dois discos em cada ciclo. Se um disco não for tocado, deve ser acrescentada uma batida suplementar, de maneira a atingir os 25 ciclos requeridos (no total são realizadas 50 batidas nos discos). A pontuação final do teste deve ser registada em tempo (décimos de segundos). ${ }^{21}$

Força de Preensão Palmar - A força de preensão manual ( $\mathrm{kg} /$ força) é um biormarcador importante de funcionalidade na pessoa idosa 22 e foi aferida usando o dinamómetro Kern Map (Versão 1.2). O procedimento para mensuração da força de preensão palmar consistiu na implementação de uma metodologia standard em que o idoso deve permanecer na posição sentado, com os pés apoiados no solo e o membro superior a ser testado mantido com o cotovelo em flexão de $90^{\circ}$, ombro em adução e antebraço na posição neutra. Para cada idoso, foram realizadas três preensões máximas, com duração de cinco segundos, intercaladas e foi registada a melhor performance. Foram coletados valores em ambas as mãos.

Avaliação das percepções do idoso sobre o trabalho com o Jogo das Mãos TA!TI! - Pela importância das medidas de autorrelato ${ }^{23}$, os autores do presente estudo desenvolveram uma lista breve de critérios que consideraram mais relevantes para apreciação pelo idosos participantes, tendo em conta os domínios abordados pelos Jogo das Mãos TA!TI!. No momento de avaliação final (T2) os idosos participantes avaliaram 6 afirmações sobre as suas percepçãos em relação à experiência do jogo, nomeadamente sobre as competências que trabalharam (ex., atividades importantes para a minha saúde; atividades importantes para o meu bem-estar emocional), as dificuldades sentidas (ex., consigo identificar atividades em que me senti mais capaz) e a importância deste tipo de atividades (ex., senti que trabalhei a agilidade dos meus braços). A cada uma das afirmações atribuíram a classificação de 0 (não concordo) a 5 (concordo totalmente). Este tipo de avaliação é uma forma de monitorizar a qualidade e o envolvimento dos jogadores nestas atividades.

\section{Análise Estatística}

A amostra em estudo foi caracterizada quanto às suas variáveis sociodemográficas, calculando valores de frequências e percentagens. Foram ainda calculados valores médios e desvio padrão $(x \pm D P)$ para as variáveis de caracterização cognitiva $(6 \mathrm{CIT})$ e para as questões sobre as percepções dos idosos durante o treino com o Jogo das Mãos TA!TI!. Para análise da evolução na coordenação dos membros superiores e na Força de Preensão Palmar entre T0 e T1 e entre T1 e T2, foi calculada a magnitude das diferenças e a sua diferença estatística foi testada por meio do teste de Wilcoxon $(p<0.05)$.

\section{Resultados}

Foram elegíveis para o estudo 23 idosos, por satisfazerem os critérios de inclusão. Da totalidade da amostra elegível, foram apenas incluídos 10 idosos cujo envolvimento no estudo não impedia a participação em outras atividades de importância na comunidade ou na instituição. Destes 10 idosos incluídos, todos terminaram o acompanhamento ao longo do estudo.

\section{Caracterização sociodemográfica e cognitiva da amostra}

A amostra deste estudo é constituída por 10 indivíduos, dos quais 9 são do sexo feminino. A maior parte dos sujeitos é viúvo/a (70.0\%) e sabe ler e escrever sem possuir grau de ensino (40.0\%) ou possui o ensino básico primário (40.0\%) (Tabela 3). A idade varia entre 81 e 92 anos, com uma média de idade de 86.70 \pm 3.43 anos. 
Tabela 3. Caracterização da amostra relativamente ao sexo, estado civil e grau de escolaridade

\begin{tabular}{cccc}
\hline & & Frequência & Percentagem válida (\%) \\
\hline \multirow{2}{*}{ Sexo } & Feminino & 9 & 90.0 \\
& Masculino & 1 & 10.0 \\
Estado Civil & Casado/a & 1 & 10.0 \\
& Viúvo/a & 7 & 70.0 \\
Escolaridade & Divorciado/a & 2 & 20.0 \\
& Sabe ler e escrever sem possuir grau de ensino & 4 & 40.0 \\
& Ensino médio & 4 & 40.0 \\
\hline
\end{tabular}

Fonte: Os autores (2021).

A pontuação total no 6 CIT é de $4.00 \pm 2.49$ nos idosos participantes. De acordo com os critérios de inclusão/exclusão previstos, estes resultados representam um perfil preservado de funcionamento cognitivo. Os itens 5 e 6 (atenção e memória) evidenciam mais erros (Tabela 4).

Tabela 4. 6CIT: pontuação total e por item, para cada participante, Média e Desvio Padrão

\begin{tabular}{llllllll}
\hline $\begin{array}{l}6 \text { CIT } \\
\text { Item }\end{array}$ & 1 & 2 & 3 & 4 & 5 & 6 & Total \\
\hline P 1 & 4 & 0 & 0 & 0 & 0 & 0 & 4 \\
P 2 & 4 & 0 & 0 & 0 & 2 & 0 & 6 \\
P 3 & 0 & 0 & 0 & 0 & 0 & 6 & 6 \\
P 4 & 0 & 0 & 0 & 0 & 4 & 2 & 0 \\
P 5 & 0 & 0 & 0 & 0 & 0 & 0 & 6 \\
P6 & 0 & 0 & 0 & 0 & 2 & 0 & 6 \\
P 7 & 0 & 0 & 0 & 0 & 2 & 4 & 0 \\
P 8 & 0 & 0 & 0 & 0 & 2 & 4 & 0 \\
P 9 & 0 & 0 & 0 & 0 & 0 & 0 & $4.00 \pm 2.49$ \\
P 10 & 0 & 0 & 0 & 0 & 4 & 0 & \\
X DP & & & & & & & 0 \\
\hline
\end{tabular}

Fonte: Os autores (2021).

\section{Evolução da coordenação dos membros superiores}

$\mathrm{Na}$ análise dos resultados da bateria EUROFIT, pode-se constatar que no período de tratamento convencional (T1-T0) não se evidenciam diferenças significativas. Por comparação, apenas durante o período de implementação do tratamento convencional com o Jogo das Mãos TA!TI! (T2-T1), foram evidentes melhorias estatisticamente significativas $(p<0,01)$ na velocidade de execução do teste (Tabela 5; Gráfico 1). 
Tabela 5. Caracterização e comparação da evolução dos resultados da bateria EUROFIT entre T0 e T1 (Tratamento Convencional) e entre T1 e T2 (Tratamento Convencional e Jogo das Mãos TA!TI!)

\begin{tabular}{lrrrrr}
\hline EUROFIT & T0 & T1 & T2 & $\begin{array}{c}\text { T1-T0 } \\
\text { Intervenção } \\
\text { Convencional }\end{array}$ & $\begin{array}{c}\text { T2-T1 } \\
\text { Intervenção } \\
\text { Convencional+Jogo }\end{array}$ \\
\hline P1 & 321 & 322 & 247 & +001 & -75 \\
P2 & 601 & 501 & 409 & -100 & -92 \\
P3 & 813 & 680 & 444 & -133 & -236 \\
P4 & 431 & 445 & 398 & +014 & -47 \\
P5 & 568 & 544 & 446 & -024 & -98 \\
P6 & 605 & 554 & 441 & -051 & -113 \\
P7 & 1167 & 1211 & 1043 & +044 & -168 \\
P8 & 494 & 494 & 380 & 000 & -114 \\
P9 & 322 & 297 & 224 & -025 & -73 \\
P10 & 418 & 401 & 302 & -017 & -99 \\
p-value & & & & 0.11 & $0.005 * *$ \\
\hline
\end{tabular}

Wilcoxon Signed Ranks Test; * $p<0.05 ; * * p<0.01$.

Fonte: Os autores (2021).

Gráfico 1. Comparação da evolução dos resultados da bateria EUROFIT entre T0 e T1 (Tratamento Convencional) e entre T1 e T2 (Tratamento Convencional e Jogo das Mãos TA!TI!)

\section{EUROFIT}

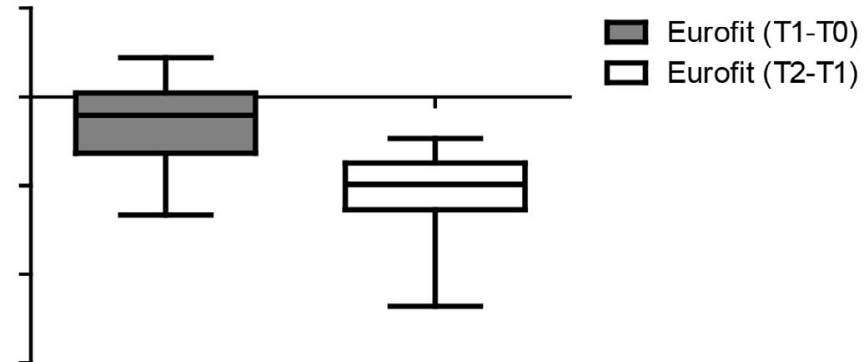

Fonte: Os autores (2021).

\section{Evolução da Força de Preensão Manual}

Analisando o efeito do tratamento convencional (T1-T0), verifica-se que os resultados da FPM não evidenciam alterações estatisticamente significativas, em qualquer dos membros superiores. No entanto, comparando estes dados com os dados relativos ao período de tempo em que ocorreu o tratamento convencional e o Jogo das Mãos TA!TI! (T2-T1), foram encontradas diferenças estatisticamente significativas para o MS direito $(p<0,01)$ e para o MS esquerdo, em 90\% dos participantes $(p<0,01)$ (Tabela 6; Gráficos 2 e 3). 
Gráfico 2. Comparação da evolução dos resultados da força de preensão manual entre T0 e T1 (Tratamento Convencional) e entre T1 e T2 (Tratamento Convencional e Jogo das Mãos TA!TI!i), no Membro Superior Esquerdo. - a escala da figura deve corresponder aos valores da FPM

\section{Força Preensão (MS Esquerdo)}

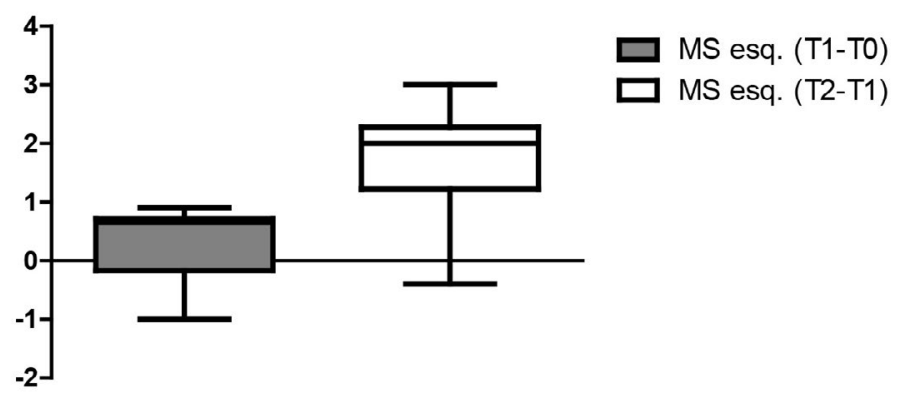

MSE, Membro Superior Esquerdo

Fonte: Os autores (2021).

Gráfico 3. Comparação da evolução dos resultados da força de preensão manual entre T0 e T1 (Tratamento Convencional) e entre T1 e T2 (Tratamento Convencional e Jogo das Mãos TA!TI! ), no Membro Superior Direito

Força Preensão (MS Direito)

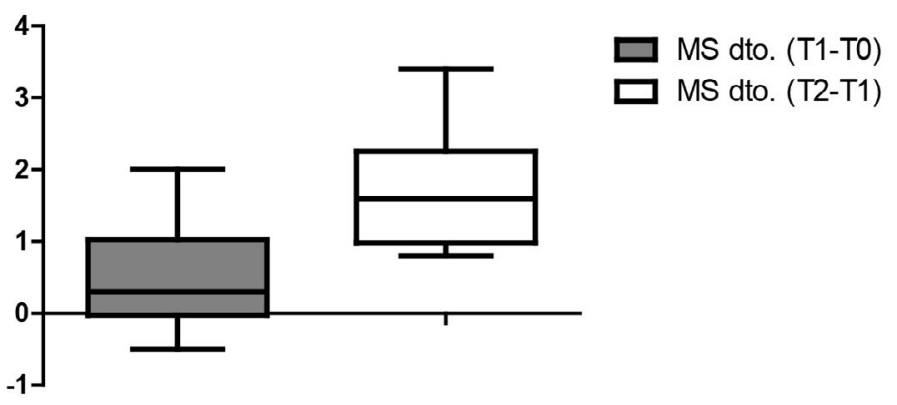

MSD, Membro Superior Direito

Fonte: Os autores (2021) 
Tabela 6. Caracterização da evolução dos resultados da força de preensão manual entre T0 e T1 (Tratamento Convencional) e entre T1 e T2 (Tratamento Convencional e Jogo das Mãos TA!T!!)

\begin{tabular}{|c|c|c|c|c|c|c|c|c|c|c|}
\hline \multirow[t]{2}{*}{$\begin{array}{r}\text { Força } \\
\text { Preensão } \\
\text { manual }\end{array}$} & \multicolumn{2}{|c|}{ TO } & \multicolumn{2}{|c|}{ T1 } & \multicolumn{2}{|c|}{ T2 } & \multicolumn{2}{|c|}{$\begin{array}{c}\text { T1-T0 } \\
\text { Intervenção Convencional }\end{array}$} & \multicolumn{2}{|c|}{$\begin{array}{c}\text { T2-T1 } \\
\text { Intervenção } \\
\text { Convencional+Jogo }\end{array}$} \\
\hline & MS Direito & MS Esquerdo & MS Direito & MS Esquerdo & MS Direito & $\begin{array}{r}\text { MS } \\
\text { Esquerdo }\end{array}$ & MS Direito & MS Esquerdo & MS Direito & MS Esquerdo \\
\hline P1 & 174 & 15,7 & 17,9 & 16,5 & 21,3 & 18,4 & 0,5 & 0,8 & 3,4 & 1,9 \\
\hline P2 & 147 & 9,6 & 14,7 & 10,2 & 15,6 & 11,2 & 0.0 & 0,6 & 0,9 & 1 \\
\hline P3 & 154 & 17,1 & 15,3 & 16,7 & 17,7 & 18,0 & $-0,1$ & $-0,4$ & 2,4 & 1,3 \\
\hline P4 & 94 & 6,0 & 9,4 & 5,9 & 10,4 & 8,1 & 0 & $-0,1$ & 1 & 2,2 \\
\hline P5 & 15,1 & 15,6 & 16,5 & 16,0 & 17,5 & 18,1 & 1,4 & 0,4 & 1 & 2,1 \\
\hline P6 & 11,2 & 7,5 & 12,1 & 8,2 & 13,4 & 11,2 & 0,9 & 0,7 & 1,3 & 3 \\
\hline P7 & 5,5 & 5,5 & 5,6 & 6,2 & 6,4 & 5,8 & 0,1 & 0,7 & 0,8 & $-0,4$ \\
\hline P8 & 12,5 & 12,8 & 13,2 & 13,7 & 15,2 & 15,8 & 0,7 & 0,9 & 2 & 2,1 \\
\hline P9 & 20,0 & 16,2 & 19,5 & 15,2 & 21,7 & 17,7 & $-0,5$ & -1 & 2,2 & 2,5 \\
\hline P10 & 19,5 & 15,5 & 21,5 & 16,2 & 23,4 & 17,6 & 2 & 0,7 & 1,9 & 1,4 \\
\hline$p$-value & & & & & & & 0.07 & 0.15 & $0.005^{* \star}$ & $0.007^{* \star}$ \\
\hline
\end{tabular}

Wilcoxon Signed Ranks Test; * $p<0.05 ; * * p<0.01$.

MSE, membro superior esquerdo

MSD, membro superior direito

Fonte: Os autores (2021) 


\section{Percepção do idoso sobre o trabalho com o Jogo das Mãos TA!TI!}

Os valores médios mais elevados foram identificados em questões sobre a importância de: jogar em grupo (4.0 \pm 0.83$)$, jogar para trabalhar raciocínio e pensamento $(4.4 \pm 0.49)$ e de jogar para treinar a agilidade dos membros superiores (4.1 \pm 0.83$)$ e atividades importantes para a minha saúde $(4.0 \pm 0.77)$.

Tabela 7. Valores de perceção dos idosos sobre a experiência com o Jogo das Mãos TATI

\begin{tabular}{|c|c|c|c|c|c|c|}
\hline & $\begin{array}{l}\text { Atividades } \\
\text { importantes } \\
\text { para a } \\
\text { minha } \\
\text { saúde }\end{array}$ & $\begin{array}{l}\text { Atividades } \\
\text { importantes } \\
\text { para o meu } \\
\text { bem-estar } \\
\text { emocional }\end{array}$ & $\begin{array}{l}\text { Consigo } \\
\text { identificar } \\
\text { atividades em } \\
\text { que me senti } \\
\text { mais capaz }\end{array}$ & $\begin{array}{l}\text { Senti que } \\
\text { trabalhei as } \\
\text { minhas } \\
\text { capacidades de } \\
\text { raciocínio e de } \\
\text { pensamento }\end{array}$ & $\begin{array}{l}\text { Senti que } \\
\text { trabalhei a } \\
\text { agilidade } \\
\text { dos meus } \\
\text { braços }\end{array}$ & $\begin{array}{l}\text { Percebi a } \\
\text { importância } \\
\text { de jogar em } \\
\text { grupo }\end{array}$ \\
\hline P1 & 4 & 5 & 3 & 4 & 4 & 5 \\
\hline P2 & 4 & 2 & 4 & 5 & 5 & 3 \\
\hline P3 & 4 & 3 & 3 & 5 & 5 & 4 \\
\hline P4 & 3 & 2 & 0 & 4 & 3 & 4 \\
\hline P5 & 3 & 1 & 1 & 4 & 3 & 3 \\
\hline P6 & 5 & 4 & 4 & 5 & 5 & 5 \\
\hline P7 & 3 & 3 & 0 & 4 & 3 & 3 \\
\hline P8 & 5 & 3 & 3 & 4 & 4 & 4 \\
\hline P9 & 4 & 3 & 0 & 4 & 4 & 5 \\
\hline P10 & 5 & 3 & 4 & 5 & 5 & 5 \\
\hline \multirow{2}{*}{$\begin{array}{l}\text { Média } \pm \\
\text { Desvio } \\
\text { padrão }\end{array}$} & 4.00 & 2,90 & 2,20 & 4,40 & 4,10 & 4.00 \\
\hline & 0,77 & 1,04 & 1,66 & 0,49 & 0,83 & 0,83 \\
\hline
\end{tabular}

Fonte: Os autores (2021).

\section{Discussão}

Os dados deste estudo piloto parecem corroborar o potencial de um jogo de tabuleiro como estratégia complementar ao tratamento convencional para o treino da coordenação dos membros superiores e da FPM, em idosos institucionalizados.

Considerando os resultados obtidos, não se verificaram alterações estatisticamente significativas na velocidade de execução do teste de toque de discos da bateria de testes físicos EUROFIT e nos resultados da FPM, durante o período de tempo em que os idosos participantes apenas foram sujeitos a tratamento convencional. No entanto, foram encontradas melhorias estatisticamente significativas na combinação do tratamento convencional e do Jogo das Mãos TA!TI!.

Estes dados obtidos com a implementação de jogos de tabuleiro como estratégia adicional à reabilitação convencional parecem indicar o potencial do jogo como complemento às terapias no idoso, especificamente para meIhoria da função dos membros superiores. O potencial do jogo na melhoria da função dos membros superiores evidenciado no presente estudo parece ser concordante com dados já relatados por vários estudos anteriores, em específico, aqueles com foco na utilização de jogos digitais, de realidade virtual e gameterapia na estimulação funcional do idoso. Por exemplo, no estudo de Sánchez-Herrera-Baeza et al. ${ }^{24}$ os autores incluíram 6 participantes com Doença de Parkinson, com idades entre os 69 e os 80 anos. Durante este estudo os participantes frequentaram sessões com 4 jogos sérios (jogos que associam à sua componente lúdica, um propósito sério), com o objetivo de melhorar a funcionalidade dos membros superiores. Após 18 sessões, de 30 minutos cada, observaram-se melhorias na FPM e na coordenação e velocidade dos membros superiores. Alguns participantes melhoraram nas atividades de vida diária (AVDs), como comer, manipular utensílios e comprar alimentos. $\underline{24}$ 
Em outro estudo de Ma e Bechkoum ${ }^{25}$, foram incluídos 8 participantes, com quadro clínico de Acidente Vascular Cerebral (AVC). Os participantes foram divididos em 2 grupos: um grupo recebeu tratamento funcional, utilizando realidade virtual, combinado com jogos sérios; o outro grupo recebeu apenas tratamento funcional, utilizando realidade virtual. Após 10 sessões, todos os participantes tiveram melhorias significativas na força, coordenação, destreza e funcionalidade dos membros superiores. No entanto, o tratamento no primeiro grupo mostrou-se mais eficaz, tendo maior impacto na recuperação da funcionalidade dos membros superiores. .25

Este efeito positivo dos jogos digitais na estimulação da função dos membros superiores em pessoas idosas foi também comprovado por uma meta-análise (61 estudos) que fez a comparação direta com a utilização de tratamentos convencionais. ${ }^{26} \mathrm{Em}$ todos os artigos referidos, não existe uma descrição detalhada das estratégias usadas no tratamento convencional e a variabilidade do tempo e frequência de intervenção é muito elevada ( $30 \mathrm{~min} / \mathrm{dia}-5 \mathrm{~h} / \mathrm{dia} ; 4$ - 12 semanas). Numa análise global ao fator de intensidade e frequência, o presente estudo apresenta um protocolo de implementação do jogo de $2 \mathrm{~h} /$ semana, durante apenas 2 semanas. Assim, a presente investigação evidencia bons resultados com a implementação do jogo de tabuleiro em níveis de intensidade bem mais baixos do que a maioria dos jogos digitais incluídos na meta-análise de Tăut et al. ${ }^{26} \mathrm{~A}$ ausência de estudos de referência nesta área sobre a utilização de jogos de tabuleiro, quando comparada com a diversidade de estudos que utilizam jogos digitais, dificulta comparações entre resultados. Até o momento, a grande maioria dos estudos relacionados explora o benefício da utilização de jogos na independência em atividades da vida diária (AVD's). $24-26$ Futuros estudos sobre a implementação dos jogos de tabuleiro, poderiam incluir não apenas a melhoria na coordenação dos membros superiores e FPM, mas também a sua relação com a a funcionalidade diária do idoso.

No presente estudo, as percepçãos dos idosos sobre a experiência do jogo revelaram-se mais importantes para critérios como jogar em grupo $(4.0 \pm 0.83)$, jogar para trabalhar raciocínio e pensamento $(4.4 \pm 0.49)$, jogar para treinar a agilidade dos membros superiores (4.1 \pm 0.83$)$, considerando-se ainda uma ativi-

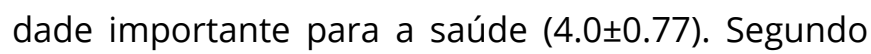
Barbosa et al. ${ }^{27}$ os fatores como a socialização com outras pessoas e a possibilidade de realizar exercício mental associado ao exercício físico, são importantes motivações para os idosos aderirem a intervenções com jogos sérios. ${ }^{27}$ Numa revisão narrativa sobre a importância de desenhar sessões de fisioterapia em contexto lúdico, os autores Barbosa et al..27 explicam a contribuição destas abordagens para melhorar a adesão e os resultados obtidos no processo de reabilitação geriátrica. Para idosos em contexto institucional, a implementação destas ferramentas pode ajudar a melhorar as interações pessoais, podendo ser descrito como um contexto ótimo para gerar cooperação entre residentes, nomeadamente entre aqueles com maior assimetria nos seus perfis funcionais, permitindo adaptar-se a dinâmicas em grupo. $\underline{28}$ Nas duplas de jogadores formadas no presente estudo, foram percebidas algumas assimetrias no perfil cognitivo (por exemplo, entre P9 e P10), no entanto, a experiência com o jogo foi ainda assim percebida como bastante positiva por ambos os jogadores. Isto pode ser indicativo do benefício de jogo de tabuleiro como um contexto propício ao envolvimento positivo em tarefas de grupo, mesmo em idosos com diferentes níveis de incapacidade.

Considerado o potencial do jogo de tabuleiro como uma ferramenta de estímulo de coordenação dos membros superiores e FPM na população idosa institucionalizada, assim como uma experiência positiva e motivadora para os participantes, devem considerar-se algumas limitações metodológicas importantes ao presente estudo. A escolha de um desenho de estudo quase-experimental permitiu aos investigadores compreender uma ação em seguida da outra em um mesmo grupo, sem controle (como fisioterapia convencional no primeiro momento e, depois fisioterapia mais o jogo de tabuleiro no segundo momento). No entanto é importante compreender que a força e coordenação são habilidades que, com a repetição e o tempo, podem ser aprimoradas. Na ausência de uma metodologia com grupo controle e aleatorização, considera-se como uma vantagem o controle de um período de tempo igual entre T0-T1 e T1-T2, que permitiu ainda assim concluir que a evolução nas variáveis em estudo entre os dois períodos de tempo foi distinta. A amostra em estudo é pouco representativa das características da população geriátrica, tanto no que diz respeito ao pequeno número de idosos incluídos, como ao contexto em que se encontram (idosos institucionalizados). Contudo, a dimensão da amostra adequa-se a um desenho de estudo piloto. Como discutido anteriormente, não foram incluídas medidas de independência funcional, o que impede 
de aferir conclusões sobre a relevância clínica do jogo em parâmetros como a independência na realização das AVD's. É importante ainda considerar a limitação no desenho da escala de avaliação das percepçãos dos idosos sobre o jogo. Esta é uma escala que considera apenas afirmações positivas, que podem não permitir um nível real de reflexão aconselhado. Por último, não ocorreu neste estudo um intervalo de tempo entre a terapia convencional e a terapia convencional em conjunto com o jogo de tabuleiro. No conjunto, estas limitações devem ser alvo de melhoria de desenho metodológico em futuras investigações nesta área.

Apesar dos resultados positivos demonstrados neste estudo, mais investigação sobre este tema é necessário, sendo relevante a inclusão de amostras mais amplas e mais heterogéneas, por exemplo, incluindo sujeitos da comunidade e incluindo grupos de idosos com declínio e/ou deterioração cognitiva.

\section{Conclusão}

Os dados exploratórios obtidos indicam que o jogo de tabuleiro é uma potencial ferramenta de estimulação geriátrica para melhorar a coordenação de membros superiores e a força de preensão manual, sendo a experiência bem percebida pelos idosos participantes.

\section{Contribuições dos autores}

Marinho $\mathrm{R}$ participou no recrutamento da amostra, na implementação do programa de intervenção e na recolha de dados ao longo do estudo. Rosa MN participou no desenho da metodologia do estudo, no tratamento e análise estatística dos dados, assim como na escrita científica do documento. Gordo S participou na redação e revisão da escrita do artigo. Pocinho $\mathrm{R}$ participou no desenho da metodologia e na redação da escrita do artigo.

\section{Conflitos de interesses}

Nenhum conflito financeiro, legal ou político envolvendo terceiros (governo, empresas e fundações privadas, etc.) foi declarado para nenhum aspecto do trabalho submetido (incluindo, mas não se limitando a subvenções e financiamentos, participação em conselho consultivo, desenho de estudo, preparação de manuscrito, análise estatística, etc.).

\section{Referências}

1. Eurostat. Population age structure indicators, 1 January 2020 (\%) [Internet]. 2013. Disponível em: https://ec.europa.eu/ eurostat/statistics-explained/index.php?title=File:Population_age_ structure_indicators,_1」January_2020_(\%25).png

2. João Neto M, Corte-Real J. The Elder institutionalized: Depression and social support. J Aging Innovation [Internet]. 2013;2(3). Disponível em: http://journalofagingandinnovation.org/ pt/volume2-edicao3-julho2013/a-pessoa-institucionalizada/

3. Instituto Nacional de Estatística. Projeções da População Residente em Portugal. Ine; 2017. Disponível em: https://www.ine.pt/xportal/ xmain?xpid=INE\&xpgid=ine destaques\&DESTAQUESdest boui $=277695619 \& D E S T A Q U E S m o d o=2$

4. Krevers B, Närväanen A-L, Öberg B. Patient evaluation of the care and rehabilitation process in geriatric hospital care. Disabil Rehabil. 2002;24(9):482-91. https://doi. org/10.1080/09638280110105268

5. Forster A, Lambley R, Young JB. Is physical rehabilitation for older people in long-term care effective? Findings from a systematic review. Age Ageing. 2010;39(2):169-75. https://doi. org/10.1093/ageing/afp247

6. Avers D. Exercise and Physical Activity for Older Adults. In: Avers D, Wong R, editores. Guccione's Geriatric Physical Therapy. 4a ed. St. Louis (MO): Mosby; 2020. p. 166-200. https://doi.org/10.1016/ B978-0-323-60912-8.00008-7

7. Burke JW, McNeill MDJ, Charles DK, Morrow PJ, Crosbie JH, McDonough SM. Optimising engagement for stroke rehabilitation using serious games. Vis Comput. 2009;25(12):1085. https://doi. org/10.1007/s00371-009-0387-4

8. Ching-Teng Y. Effect of board game activities on cognitive function improvement among older adults in adult day care centers. Soc Work Health Care. 2019;58(9):825-38. https://doi.org/ 10.1080/00981389.2019.1656143

9. Koivisto J, Malik A. Gamification for Older Adults: A Systematic Literature Review. Gerontologist. 2020;61(7): e360-e372. https:// doi.org/10.1093/geront/gnaa047

10. Gauthier A, Kato PM, Bul KCM, Dunwell I, Walker-Clarke A, Lameras P. Board Games for Health: A Systematic Literature Review and Meta-Analysis. Games Health J. 2018;8(2):85-100. https://doi.org/10.1089/g4h.2018.0017

11. Ibrahim K, May CR, Patel HP, Baxter M, Sayer AA, Roberts HC. Implementation of grip strength measurement in medicine for older people wards as part of routine admission assessment: identifying facilitators and barriers using a theory-led intervention. BMC Geriatr. 2018;18(1):79. https://doi.org/10.1186/s12877-018$\underline{0768-5}$ 
12. Woytowicz E, Whitall J, Westlake KP. Age-related changes in bilateral upper extremity coordination. Curr Geriatr reports [Internet]. 2016/07/02. 2016 Sep;5(3):191-9. Available from: https://pubmed.ncbi.nlm.nih.gov/27917365

13. Altschul DM, Deary IJ. Playing Analog Games Is Associated With Reduced Declines in Cognitive Function: A 68-Year Longitudinal Cohort Study. Journals Gerontol Ser B. 2020;75(3):474-82. https:// doi.org/10.1093/geronb/gbz149

14. Dartigues JF, Foubert-Samier A, Le Goff M, Viltard M, Amieva $\mathrm{H}$, Orgogozo JM, et al. Playing board games, cognitive decline and dementia: a French population-based cohort study. BMJ Open. 2013;3(8):e002998. http://dx.doi.org/10.1136/ bmjopen-2013-002998

15. Rodríguez-Fórtiz MJ, Rodríguez-Domínguez C, Cano $P$, Revelles J, Rodríguez-Almendros ML, Hurtado-Torres MV, et al. Serious games for the cognitive stimulation of elderly people. In: 2016 IEEE International Conference on Serious Games and Applications for Health (SeGAH); 2016. https://doi.org/10.1109/ SeGAH.2016.7586261

16. Mortenson WB, Sixsmith A, Kaufman D. Non-Digital Game Playing by Older Adults. Can J Aging. 2017;36(3):342-50. https:// doi.org/10.1017/s0714980817000162

17. Rosa M, Marinho R, Gordo S, Pocinho R. El juego como sistema de evaluación en ancianos institucionalizados - estudio piloto. Retos. 2021;43:370-8. https://doi.org/10.47197/retos.v43i0.89551

18. Weissenstein A, Ligges S, Brouwer B, Marschall B, Friederichs $\mathrm{H}$. Measuring the ambiguity tolerance of medical students: a cross-sectional study from the first to sixth academic years. BMC Fam Pract. 2014;15:6. https://doi.org/10.1186/1471-2296-15-6

19. Brooke P, Bullock R. Validation of a 6 item cognitive impairment test with a view to primary care usage. Int J Geriatr Psychiatry. 1999;14(11):936-40. Citado em: PMID: 10556864

20. Apóstolo JLA, Paiva DS, Silva RCG, Santos EJF, Schultz TJ. Adaptation and validation into Portuguese language of the six-item cognitive impairment test (6CIT). Aging Ment Health. 2018;22(9):1190-5. https://doi.org/10.1080/13607863.2017.1348 473
21. Silva LM, Aidar FJ, Matos DG, Silva LM, Aidar FJ, Matos DG, et al. Validation of automated apparatus for upper limb velocity testing. Motricidade. 2018;14(4):86-93. https://doi.org/10.6063/ motricidade. 15983

22. Bohannon RW. Grip Strength: An Indispensable Biomarker For Older Adults. Clin Interv Aging. 2019;14:1681-91. https://doi. org/10.2147/cia.s194543

23. Krogsgaard MR, Brodersen J, Christensen KB, Siersma V, Kreiner S, Jensen J, et al. What is a PROM and why do we need it? Scand J Med Sci Sports. 2021;31(5):967-71. Available from: https:// doi.org/10.1111/sms.13892

24. Sánchez-Herrera-Baeza P, Cano-de-la-Cuerda R, Oña-Simbaña ED, Palacios-Ceña D, Pérez-Corrales J, Cuenca-Zaldivar JN, et al. The Impact of a Novel Immersive Virtual Reality Technology Associated with Serious Games in Parkinson's Disease Patients on Upper Limb Rehabilitation: A Mixed Methods Intervention Study. Sensors. 2020;20(8):2168. https://doi.org/10.3390/s20082168

25. Ma M, Bechkoum K. Serious games for movement therapy after stroke. 2008 IEEE Int Conf Syst Man Cybern. 2008;1872-7. http://dx.doi.org/10.1109/ICSMC.2008.4811562

26. Tăut $D$, Pintea S, Roovers J-PWR, Mañanas M-A, Băban A. Play seriously: Effectiveness of serious games and their features in motor rehabilitation. A meta-analysis. NeuroRehabilitation. 2017;41(1):105-18. https://doi.org/10.3233/nre-171462

27. Barbosa H, Castro A, Carrapatoso E. Serious Games and Rehabilitation for Elderly Adults. Glob Sci J [Internet]. 2018;6(1):275-83. https://www.globalscientificjournal.com/ researchpaper/Serious-Games-and-Rehabilitation-for-ElderlyAdults.pdf

28. Noda S, Shirotsuki K, Nakao M. The effectiveness of intervention with board games: a systematic review. Biopsychosoc Med. 2019;13:22. https://dx.doi. org/10.1186\%2Fs13030-019-0164-1 